\title{
U.S.D.A., Sedimentation Laboratory, Oxford, Mississippi
}

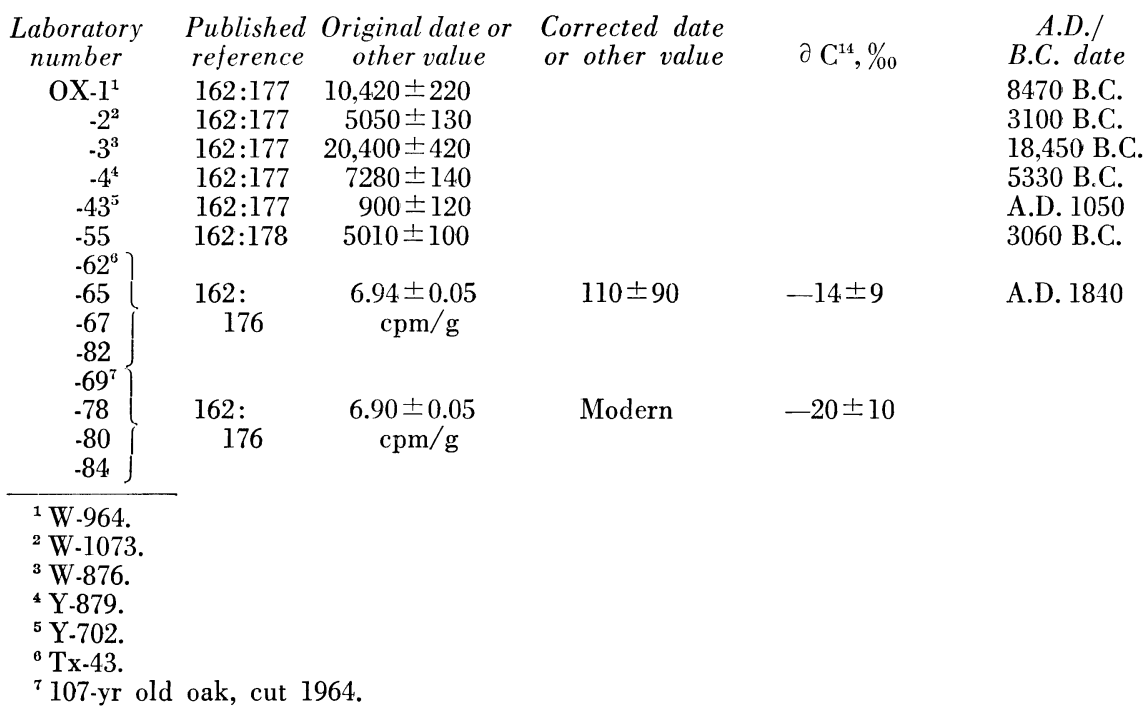

\section{University of Pennsylvania Applied Science Center for Archaeology}

\begin{tabular}{|c|c|c|c|c|}
\hline $\begin{array}{l}\text { Laboratory } \\
\text { number }\end{array}$ & $\begin{array}{l}\text { Published } \\
\text { reference }\end{array}$ & $\begin{array}{cl}\text { Original daie or } & \text { Corrected date } \\
\text { other value } & \text { or other value }\end{array}$ & $\partial \mathrm{C}^{14}, \% 0$ & $\begin{array}{l}\text { A.D./ } \\
\text { B.C. date }\end{array}$ \\
\hline P.11 & $62: 47$ & $3710 \pm 98$ & & 1752 B.C. \\
\hline$-11^{1}$ & $59: 185$ & $\mathrm{C}_{\mathrm{innm}}+23.4 \pm 2.5$ & & \\
\hline-12 & $60: 151$ & $9190 \pm 590^{\mathrm{s}}$ & & 7235 B.C. \\
\hline-13 & $63: 11$ & $2213 \pm 110$ & & 255 B.C. \\
\hline-14 & $60: 150$ & $2880 \pm 340^{\mathrm{s}}$ & & 1455 B.C. \\
\hline$-14 a$ & $60: 150$ & $2485 \pm 235^{\mathrm{s}}$ & & 530 B.C. \\
\hline$-15 a$ & $60: 150$ & $2775 \pm 315^{\mathrm{s}}$ & & 820 B.C. \\
\hline-16 & $63: 4$ & $780 \pm 150^{s}$ & & A.D. 1177 \\
\hline-17 & $60: 150$ & $2975 \pm 345^{\mathrm{s}}$ & & 1020 B.C. \\
\hline$-17 a$ & $60: 150$ & $2650 \pm 340^{\mathrm{s}}$ & & 695 B.C. \\
\hline-18 & $60: 150$ & $1220 \pm 230^{\mathrm{s}}$ & & A.D. 775 \\
\hline-19 & $60: 150$ & $7015 \pm 405^{s}$ & & 5060 B.C. \\
\hline$-19 a$ & $60: 150$ & $7395 \pm 495^{\mathrm{s}}$ & & 5440 B.C. \\
\hline$-19 b$ & $60: 150$ & $7430 \pm 460^{s}$ & & 5475 B.C. \\
\hline-20 & $60: 150$ & $11,400 \pm 800^{\mathrm{s}}$ & & 9445 B.C. \\
\hline$-20 \mathrm{~b}$ & $60: 150$ & $11,550 \pm 750^{\mathrm{s}}$ & & 9595 B.C. \\
\hline-24 & $60: 150$ & $8785 \pm 575^{s}$ & & 6830 B.C. \\
\hline$-24 a$ & $60: 150$ & $8360 \pm 510^{s}$ & & 6405 B.C. \\
\hline-26 & $60: 150$ & $7680 \pm 470^{s}$ & & 5725 B.C. \\
\hline$-26 a$ & $60: 150$ & $7905 \pm 475^{s}$ & & 5950 B.C. \\
\hline-27 & $60: 150$ & $12,275 \pm 825^{\mathrm{s}}$ & & 10,320 B.C. \\
\hline-29 & $63: 4$ & $720 \pm 200^{\mathrm{s}}$ & & A.D. 1237 \\
\hline$-30^{1}$ & $59: 185$ & $\mathrm{C}_{\mathrm{anom}}+6.9 \pm 3.8$ & & \\
\hline-30 & $64 a: 362$ & $2420 \pm 51$ & & 470 B.C. \\
\hline-31 & $63: 4$ & $820 \pm 200^{\mathrm{s}}$ & & A.D. 1137 \\
\hline-32 & $60: 150$ & $2500 \pm 260^{\mathrm{s}}$ & & 545 B.C. \\
\hline$-32 a$ & $60: 150$ & $2890 \pm 310^{\mathrm{s}}$ & & 935 B. \\
\hline-33 & $60: 150$ & $3270 \pm 390^{\mathrm{s}}$ & & 1315 B.C. \\
\hline$-33 a$ & $60: 150$ & $2630 \pm 260^{\mathrm{s}}$ & & 675 B.C. \\
\hline-34 & $60: 150$ & $4830 \pm 480^{s}$ & & \\
\hline-35 & $60: 150$ & $4730 \pm 320^{s}$ & & \\
\hline
\end{tabular}




\begin{tabular}{|c|c|c|c|c|c|}
\hline $\begin{array}{l}\text { Laboratory } \\
\text { number }\end{array}$ & $\begin{array}{l}\text { Published } \\
\text { reference }\end{array}$ & $\begin{array}{l}\text { Original date or } \\
\quad \text { other value }\end{array}$ & $\begin{array}{l}\text { Corrected date } \\
\text { or other value }\end{array}$ & $\partial \mathrm{C}^{14}, \%$ & $\begin{array}{c}\text { A.D./ } \\
\text { B.C. date }\end{array}$ \\
\hline-36 & $60: 150$ & $6385 \pm 425^{\mathrm{s}}$ & & & 4430 B.C. \\
\hline-37 & $60: 151$ & $8070 \pm 500^{s}$ & & & 6115 B.C. \\
\hline-38 & $60: 151$ & $9220 \pm 570^{\mathrm{s}}$ & & & 7265 B.C. \\
\hline-39 & $60: 151$ & $11,860 \pm 840^{\mathrm{s}}$ & & & 9905 B.C. \\
\hline-40 & $61: 922$ & $6.40 \pm 0.19 \mathrm{cpm}^{\mathrm{s}}$ & & & \\
\hline$-40 \mathrm{a}$ & $61: 922$ & $6.34 \pm 0.15 \mathrm{cpm}^{\mathrm{s}}$ & & & \\
\hline-41 & $60: 150$ & $2890 \pm 370^{\mathrm{s}}$ & & & 935 B.C. \\
\hline$-42,42 a^{2}$ & $61: 922$ & $>25,000^{\mathrm{s}}$ & & & \\
\hline-43 & $60: 150$ & $2200 \pm 280^{s}$ & & & 245 B.C. \\
\hline-44 & $60: 150$ & $2970 \pm 270^{\mathrm{s}}$ & & & 1015 B.C. \\
\hline-45 & $60: 150$ & $6515 \pm 425^{\mathrm{s}}$ & & & 4560 B.C. \\
\hline-46 & $61: 922$ & $6.63 \pm 0.15 \mathrm{cpm}^{\mathrm{s}}$ & & & \\
\hline$-46 a$ & $61: 922$ & $6.89 \pm 0.13 \mathrm{cpm}^{\mathrm{s}}$ & & & \\
\hline-48 & $61: 922$ & 2.000 & & & 18,045 B.C. \\
\hline & & $\begin{array}{l}+.00 \quad \mathrm{~s} \\
-5000\end{array}$ & & & \\
\hline-49 & $61: 922$ & $>25,000^{\mathrm{s}}$ & & & \\
\hline$-50,50 \mathrm{a}^{3}$ & $61: 922$ & $>25,000^{\mathrm{s}}$ & & & \\
\hline-51 & $61: 922$ & $>25,000^{\circ}$ & & & \\
\hline-52 & $60: 150$ & $2860 \pm 380^{s}$ & & & 905 B.C. \\
\hline-53 & $61: 922$ & $10,580 \pm 720^{\mathrm{s}}$ & & & 8625 B.C. \\
\hline-54 & $61: 922$ & $2740 \pm 300^{\mathrm{s}}$ & & & 785 B.C. \\
\hline-55 & $63: 5$ & $1130 \pm 200^{\mathrm{s}}$ & & & A.D. 827 \\
\hline-62 & $63: 9$ & $2060 \pm 200^{s}$ & & & 102 B.C. \\
\hline-63 & $63: 6$ & $1480 \pm 240^{\mathrm{s}}$ & & & A.D. 477 \\
\hline-65 & $63: 6$ & $1320 \pm 230^{\mathrm{s}}$ & & & A.D. 637 \\
\hline-67 & $63: 5$ & $1230 \pm 240^{\mathrm{s}}$ & & & A.D. 727 \\
\hline-68 & $63: 5$ & $1350 \pm 360^{\mathrm{s}}$ & & & A.D. 607 \\
\hline-69 & $63: 6$ & $1070 \pm 210^{\mathrm{s}}$ & & & A.D. 887 \\
\hline-70 & $63: 8$ & $1420 \pm 230^{\mathrm{s}}$ & & & A.D. 537 \\
\hline-71 & $63: 7$ & $1630 \pm 230$ & & & A.D. 328 \\
\hline-73 & $63: 5$ & $1430 \pm 190^{\mathrm{s}}$ & & & A.D. 527 \\
\hline .74 & $63: 9$ & $2183 \pm 122$ & & & 225 B.C. \\
\hline-75 & $63: 9$ & $2508 \pm 130$ & & & 550 B.C. \\
\hline .76 & $63: 9$ & $2632 \pm 128$ & & & 674 B.C. \\
\hline-77 & $63: 9$ & $2191 \pm 120$ & & & 235 B.C. \\
\hline-80 & $63: 7$ & $1398 \pm 116$ & & & A.D. 560 \\
\hline-83 & $63: 6$ & $1013 \pm 111$ & & & A.D. 945 \\
\hline-84 & $63: 7$ & $1296 \pm 108$ & & & A.D. 662 \\
\hline-85 & $63: 7$ & $1002 \pm 108$ & & & A.D. 956 \\
\hline-88 & $63: 7$ & $1231 \pm 108$ & & & A.D. 727 \\
\hline-92 & $63: 6$ & $910 \pm 145$ & & & A.D. 1048 \\
\hline-93 & $63: 7$ & $1700 \pm 150$ & & & A.D. 258 \\
\hline .94 & $63: 8$ & $1429 \pm 121$ & & & A.D. 529 \\
\hline .95 & $63: 7$ & $1641 \pm 106$ & & & A.D. 317 \\
\hline-96 & $63: 10$ & $2635 \pm 125$ & & & 677 B.C. \\
\hline-97 & $63: 5$ & $1146 \pm 95$ & & & A.D. 812 \\
\hline-98 & $63: 8$ & $1619 \pm 210$ & d & & \\
\hline .99 & $64: 147$ & $2660 \pm 118$ & & & 710 B.C. \\
\hline-102 & $63: 12$ & $3310 \pm 200^{\mathrm{s}}$ & & & 1353 B.C. \\
\hline-103 & $63: 12$ & $3 \angle 80 \pm 200^{s}$ & & & 1423 B.C. \\
\hline-104 & $63: 12$ & $3000 \pm 170^{\mathrm{s}}$ & & & 1043 B.C. \\
\hline-105 & $63: 12$ & $4040 \pm 280^{s}$ & & & 2083 B.C. \\
\hline-108 & $63: 12$ & $3080 \pm 210^{s}$ & & & 1123 B.C. \\
\hline-111 & $62: 49$ & $2770 \pm 130$ & & & 812 B.C. \\
\hline .115 & $62: 56$ & $2468 \pm 133$ & & & 510 B.C. \\
\hline .116 & $62: 56$ & $2377 \pm 110$ & & & 419 B.C. \\
\hline-117 & $62: 46$ & $1937 \pm 104$ & & & A.D. 21 \\
\hline-118 & $62: 56$ & $1928 \pm 105$ & & & A.D. 30 \\
\hline-119 & $62: 55$ & $1460 \pm 200$ & & & A.D. 498 \\
\hline-120 & $62: 55$ & $1702 \pm 103$ & & & A.D. 256 \\
\hline
\end{tabular}


Comprehensive Index, 1950-1965

\begin{tabular}{|c|c|c|c|c|}
\hline $\begin{array}{l}\text { Laboratory } \\
\text { number }\end{array}$ & $\begin{array}{l}\text { Published } \\
\text { reference }\end{array}$ & $\begin{array}{l}\text { Original date or } \\
\text { other value }\end{array}$ & $\begin{array}{l}\text { Corrected date } \\
\text { or other value }\end{array}$ & $\partial \mathrm{C}^{14}, \%$ \\
\hline$-120 \mathrm{a}$ & $62: 55$ & $1226 \pm 100$ & & \\
\hline $\begin{array}{l}-121 \\
-123\end{array}$ & $\begin{array}{l}62: 55 \\
62: 55\end{array}$ & $\begin{array}{l}1423 \pm 175 \\
1817 \pm 103\end{array}$ & & \\
\hline-124 & $62: 56$ & $2291 \pm 115$ & & \\
\hline-125 & $62: 57$ & $2386 \pm 114$ & & \\
\hline-126 & $62: 57$ & $2550 \pm 116$ & & \\
\hline-127 & $62: 47$ & $2701 \pm 90$ & & \\
\hline-128 & $62: 47$ & $2631 \pm 90$ & & \\
\hline-129 & $62: 57$ & $3240 \pm 130$ & & \\
\hline-130 & $62: 50$ & $927 \pm 97$ & & \\
\hline-133 & $62: 47$ & $2939 \pm 122$ & & \\
\hline-133 & $64: 147$ & $2939 \pm 122$ & & \\
\hline$-134^{1,7}$ & $59: 185$ & $\mathrm{C}_{\text {anom }}+10.6 \pm 3.4$ & & \\
\hline-134 & $62: 47$ & $2606 \pm 117$ & & \\
\hline-135 & $62: 47$ & $2623 \pm 119$ & & \\
\hline$-135^{7}$ & $59: 185$ & See P-134 & c & \\
\hline-136 & $62: 47$ & $2690 \pm 120$ & & \\
\hline-137 & $62: 47$ & $3113 \pm 125$ & & \\
\hline-138 & $63: 9$ & $1369 \pm 102$ & d & \\
\hline-139 & $63: 9$ & $2706 \pm 118$ & $\mathrm{~d}$ & \\
\hline-14.1 & $62: 56$ & $2275 \pm 116$ & & \\
\hline-142 & $62: 56$ & $2281 \pm 113$ & & \\
\hline$-143 a$ & $62: 56$ & $2177 \pm 112$ & & \\
\hline$-143 b$ & $62: 56$ & $2318 \pm 113$ & & \\
\hline-144 & $62: 56$ & $2193 \pm 111$ & & \\
\hline-145 & $62: 57$ & $2970 \pm 120$ & & \\
\hline-146 & $62: 55$ & $949 \pm 98$ & & \\
\hline-147 & $62: 55$ & $1576 \pm 104$ & & \\
\hline-149 & $62: 55$ & $1707 \pm 93$ & & \\
\hline-150 & $62: 55$ & $1692 \pm 104$ & & \\
\hline-152 & $62: 57$ & $2101 \pm 108$ & & \\
\hline-153 & $62: 57$ & $2041 \pm 107$ & & \\
\hline-154 & $62: 57$ & $1847 \pm 106$ & & \\
\hline-155 & $62: 57$ & $2522 \pm 114$ & & \\
\hline-156 & $62: 57$ & $2962 \pm 120$ & & \\
\hline-157 & $62: 50$ & $5460 \pm 160$ & & \\
\hline-158 & $62: 54$ & $6953 \pm 181$ & & \\
\hline-160 & $62: 54$ & $1189 \pm 93$ & & \\
\hline-161 & $62: 54$ & $1159 \pm 122$ & $1159 \pm 122$ & \\
\hline-162 & $62: 53$ & $619 \pm 103$ & $619 \pm 103$ & \\
\hline-163 & $62: 53$ & $544 \pm 113$ & $544 \pm 113$ & \\
\hline-164 & $62: 52$ & $585 \pm 93$ & $585 \pm 93$ & \\
\hline-165 & $62: 54$ & $654 \pm 93$ & & \\
\hline-166 & $62: 54$ & $843 \pm 96$ & & \\
\hline$-167^{1}$ & $59: 185$ & $\mathrm{C}_{\mathrm{anom}}+26.5 \pm 5.5$ & & \\
\hline$-168^{1}$ & $59: 185$ & $\mathrm{C}_{\text {anom }}+0.4 \pm 9.4$ & & \\
\hline-169 & $62: 54$ & $850 \pm 95$ & & \\
\hline-170 & $62: 57$ & $2032 \pm 106$ & & \\
\hline-172 & $62: 57$ & $2040 \pm 109$ & & \\
\hline-174 & $63: 10$ & $1753 \pm 105$ & & \\
\hline-175 & $63: 10$ & $2244 \pm 133$ & d & \\
\hline-179 & $62: 51$ & $4161 \pm 151$ & & \\
\hline-180 & $62: 51$ & $4083 \pm 137$ & & \\
\hline-181 & $62: 52$ & $3106 \pm 122$ & & \\
\hline-183 & $62: 52$ & $1846 \pm 106$ & & \\
\hline-184 & $62: 52$ & $2545 \pm 115$ & & \\
\hline-185 & $62: 50$ & $3000 \pm 120$ & & \\
\hline-186 & $62: 49$ & $2881 \pm 119$ & & \\
\hline-187 & $62: 49$ & $2765 \pm 117$ & & \\
\hline-188 & $62: 50$ & $3981 \pm 135$ & & \\
\hline-189 & $62: 50$ & $3960 \pm 134$ & & \\
\hline
\end{tabular}

A.D./

B.C. date

A.D. 732

A.D. 535

A.D. 141

333 B.C.

428 B.C.

592 B.C.

743 B.C.

673 B.C.

1282 B.C.

A.D. 1031

981 B.C.

989 B.C.

648 B.C.

665 B.C.

732 B.C.

1155 B.C.

317 B.C.

323 B.C.

219 B.C.

360 B.C.

235 B.C.

1012 B.C.

A.D. 1009

A.D. 382

A.D. 251

A.D. 266

143 B.C.

83 B.C.

A.D. 111

564 B.C.

1004 B.C.

3502 B.C.

4995 B.C.

A.D. 769

A.D. 799

A.D. 1339

A.D. 1414

A.D. 1373

A.D. 1308

A.D. 1115

A.D. 1108

74 B.C.

82 B.C.

A.D. 205

2203 B.C.

2125 B.C.

1148 B.C.

A.D. 112

587 B.C.

1042 B.C.

923 B.C.

807 B.C.

2023 B.C.

2002 B.C. 


\begin{tabular}{|c|c|c|c|c|}
\hline $\begin{array}{l}\text { Laboratory } \\
\text { number }\end{array}$ & $\begin{array}{l}\text { Published } \\
\text { reference }\end{array}$ & $\begin{array}{l}\text { Original date or } \\
\text { other value }\end{array}$ & $\begin{array}{l}\text { Corrected date } \\
\text { or other value }\end{array}$ & $\partial \mathrm{C}^{14}, \% 0$ \\
\hline-190 & $62: 50$ & $3960 \pm 134$ & & \\
\hline-191 & $62: 50$ & $4021 \pm 134$ & & \\
\hline-192 & $63: 10$ & $1727 \pm 105$ & & \\
\hline-194 & $62: 50$ & $4115 \pm 136$ & & \\
\hline .195 & $62: 51$ & $3925 \pm 134$ & & \\
\hline-196 & $62: 51$ & $4421 \pm 141$ & & \\
\hline-198 & $62: 50$ & $3083 \pm 122$ & & \\
\hline-199 & $62: 51$ & $4400 \pm 142$ & & \\
\hline-200 & $62: 53$ & $3457 \pm 127$ & & \\
\hline-201 & $62: 53$ & $3492 \pm 128$ & & \\
\hline-202 & $62: 52$ & $3503 \pm 128$ & & \\
\hline .203 & $63: 10$ & $2646 \pm 177$ & & \\
\hline-204 & $62: 52$ & $3449 \pm 127$ & & \\
\hline-205 & $62: 52$ & $3294 \pm 125$ & & \\
\hline-206 & $62: 49$ & $1926 \pm 92$ & & \\
\hline-207 & $63: 11$ & $3958 \pm 168$ & & \\
\hline-208 & $63: 11$ & $3560 \pm 123$ & d & \\
\hline .209 & $63: 11$ & $3906 \pm 133$ & & \\
\hline-210 & $63: 11$ & $2898 \pm 136$ & d & \\
\hline-211 & $63: 11$ & $2354 \pm 135$ & $d$ & \\
\hline-212 & $63: 11$ & $2404 \pm 137$ & d & \\
\hline-213 & $63: 11$ & $2910 \pm 129$ & & \\
\hline-214 & $62: 48$ & $4447 \pm 150$ & & \\
\hline-215 & $62: 48$ & $4594 \pm 91$ & & \\
\hline-216 & $62: 48$ & $4082 \pm 102$ & & \\
\hline-217 & $62: 57$ & $1960 \pm 90$ & & \\
\hline-218 & $64: 148$ & $2900 \pm 51$ & & \\
\hline .220 & $64: 148$ & $2750 \pm 49$ & & \\
\hline-221 & $64: 147$ & $2660 \pm 62$ & & \\
\hline$-222^{1}$ & $59: 185$ & $\mathrm{C}_{\text {anom }} 0 \pm 4.5$ & & \\
\hline-222 & $64: 147$ & $2480 \pm 48$ & & \\
\hline-223 & $64: 148$ & $2830 \pm 45$ & & \\
\hline .225 & $63: 8$ & $1651 \pm 130$ & & \\
\hline$-226^{1}$ & $59: 185$ & $\mathrm{C}_{\mathrm{anom}}+10.4 \pm 4.6$ & & \\
\hline$-227^{1}$ & $59: 185$ & $\mathrm{C}_{\text {anom }}+22.3 \pm 4.6$ & & \\
\hline-227 & $62: 47$ & $3055 \pm 91$ & & \\
\hline .228 & $63: 10$ & $3208 \pm 156$ & $d$ & \\
\hline .229 & $64: 153$ & $1490 \pm 85$ & & \\
\hline-230 & $64: 153$ & $1300 \pm 93$ & & \\
\hline-232 & $64: 153$ & $1260 \pm 49$ & & \\
\hline-234 & $64: 153$ & $1260 \pm 56$ & & \\
\hline-235 & $64: 153$ & $1150 \pm 44$ & & \\
\hline-236 & $64: 153$ & $1250 \pm \leq 8$ & & \\
\hline .237 & $64: 153$ & $1260 \pm 72$ & & \\
\hline-238 & $64: 153$ & $1200 \pm 48$ & & \\
\hline-242 & $64: 153$ & $1230 \pm 48$ & & \\
\hline-243 & $64: 153$ & $1220 \pm 55$ & & \\
\hline-244 & $64: 153$ & $1210 \pm 83$ & & \\
\hline .245 & $64: 153$ & $1180 \pm 79$ & & \\
\hline-247 & $64: 153$ & $1270 \pm 49$ & & \\
\hline-248 & $64: 154$ & $1190 \pm 47$ & & \\
\hline-249 & $64: 154$ & $1210 \pm 48$ & & \\
\hline-250 & $108: 88$ & $2740 \pm 79$ & & \\
\hline-251 & $64: 153$ & $1260 \pm 110$ & & \\
\hline .254 & $64: 150$ & $3450 \pm 62$ & & \\
\hline .256 & $64: 147$ & $2510 \pm 61$ & & \\
\hline-257 & $64: 147$ & $2120 \pm 59$ & & \\
\hline$-259^{1}$ & $59: 185$ & $\mathrm{C}_{\text {anom }}+5.9 \pm 5.5$ & & \\
\hline-261 & $108: 99$ & $1263 \pm 54$ & & \\
\hline-262 & $108: 99$ & $1032 \pm 54$ & & \\
\hline$-268^{1}$ & $59: 185$ & $\mathrm{C}_{\text {anom }}+11.4 \pm 5.4$ & & \\
\hline
\end{tabular}

A.D./ B.C. date 2002 B.C. 2063 B.C. A.D. 231 2157 B.C. 1967 B.C. 2463 B.C. 1125 B.C. 2442 B.C. 1499 B.C. 1534 B.C. 1515 B.C. 688 B.C.

1491 B.C.

1336 B.C. A.D. 32 2000 B.C.

918 B.C.

952 B.C. 2489 B.C. 2636 B.C. 2124 B.C. 2 B.C.

950 B.C.

800 B.C.

710 B.C.

530 B.C. 880 B.C. A.D. 307

1097 B.C.

A.D. 460 A.D. 650

A.D. 690

A.D. 690

A.D. 800

A.D. 700

A.D. 690

A.D. 750

A.D. 720

A.D. 730

A.D. 740

A.D. 770

A.D. 680

A.D. 760

A.D. 740 790 B.C.

A.D. 690

1500 B.C. 560 B.C. 170 B.C.

A.D. 687 A.D. 918 


\begin{tabular}{|c|c|c|c|c|}
\hline $\begin{array}{l}\text { Laboratory } \\
\text { number }\end{array}$ & $\begin{array}{l}\text { Published } \\
\text { reference }\end{array}$ & $\begin{array}{c}\text { Original date or } \begin{array}{l}\text { Corrected date } \\
\text { other value }\end{array} \\
\text { or other value }\end{array}$ & $\partial \mathrm{C}^{14}, \% 0$ & $\begin{array}{l}\text { A.D./ } \\
\text { B.C. date }\end{array}$ \\
\hline-269 & $108: 100$ & $771 \pm 51$ & & A.D. 1179 \\
\hline-270 & $64: 151$ & $3340 \pm 51$ & & 1390 B.C. \\
\hline$-272^{1}$ & $59: 185$ & $\mathrm{C}_{\text {anom }}-5.0 \pm 4.1$ & & \\
\hline .273 & $64: 152$ & $3980 \pm 97$ & & 2030 B.C. \\
\hline$-274^{1}$ & $59: 185$ & $\mathrm{C}_{\mathrm{anom}}-1.3 \pm 12.2$ & & \\
\hline$-275^{1,7}$ & $59: 185$ & $\mathrm{C}_{\mathrm{anom}}+10.6 \pm 3.4$ & & \\
\hline-275 & $64: 146$ & $2560 \pm 60$ & & 610 B.C. \\
\hline-278 & $64: 155$ & $1150 \pm 47$ & & A.D. 800 \\
\hline-279 & $64: 156$ & $1180 \pm 55$ & & A.D. 770 \\
\hline-281 & $64: 156$ & $1530 \pm 52$ & & A.D. 420 \\
\hline-282 & $64: 154$ & $1310 \pm 44$ & & A.D. 640 \\
\hline$-283^{1}$ & $59: 185$ & $\mathrm{C}_{\mathrm{anom}}-5.9 \pm 5.2$ & & \\
\hline-284 & $64: 156$ & $1550 \pm 58$ & & A.D. 400 \\
\hline-285 & $64: 154$ & $1970 \pm 44$ & & 20 B.C. \\
\hline-286 & $64: 155$ & $2100 \pm 45$ & & 150 B.C. \\
\hline-287 & $64: 155$ & $2000 \pm 63$ & & 50 B.C. \\
\hline-288 & $64: 155$ & $2090 \pm 55$ & & 140 B.C. \\
\hline-289 & $64: 155$ & $2040 \pm 57$ & & 90 B.C. \\
\hline-292 & $64: 152$ & $920 \pm 44$ & & A.D. 1030 \\
\hline-293 & $64: 154$ & $1340 \pm 57$ & & A.D. 610 \\
\hline-294 & $64: 156$ & $1350 \pm 52$ & & A.D. 600 \\
\hline-295 & $64: 156$ & $1740 \pm 50$ & & A.D. 210 \\
\hline-296 & $64: 156$ & $1720 \pm 61$ & & A.D. 230 \\
\hline-297 & $64: 146$ & $4690 \pm 62$ & & 2740 B.C. \\
\hline-298 & $64: 146$ & $4960 \pm 58$ & & 3010 B.C. \\
\hline-299 & $64: 150$ & $3750 \pm 97$ & & 1800 B.C. \\
\hline-300 & $64: 150$ & $3870 \pm 61$ & & 1920 B.C. \\
\hline$-303 \mathrm{a}$ & $64: 150$ & $3750 \pm 112$ & & 1800 B.C. \\
\hline-304 & $64: 144$ & $>42,200$ & & \\
\hline-306 & $64: 149$ & $4450 \pm 75$ & & 2500 B.C. \\
\hline-307 & $64: 149$ & $4440 \pm 64$ & & 2490 B.C. \\
\hline-308 & $64: 158$ & $3750 \pm 85$ & & 1800 B.C. \\
\hline-309 & $64: 158$ & $3870 \pm 85$ & & 1920 B.C. \\
\hline-311 & $64: 158$ & $4050 \pm 85$ & & 2100 B.C. \\
\hline-312 & $64: 149$ & $3840 \pm 72$ & & 1890 B.C. \\
\hline$-313 a$ & $64: 145$ & $7350 \pm 85$ & & 5400 B.C. \\
\hline-314 & $64: 145$ & $7340 \pm 94$ & & 5390 B.C. \\
\hline-315 & $64: 146$ & $6990 \pm 121$ & & 5040 B.C. \\
\hline-316 & $64: 146$ & $7170 \pm 134$ & & 5220 B.C. \\
\hline-317 & $64: 149$ & $4210 \pm 64$ & & 2260 B.C. \\
\hline-318 & $64: 149$ & $4.070 \pm 72$ & & 2120 B.C. \\
\hline-319 & $64: 150$ & $3980 \pm 66$ & & 2030 B.C. \\
\hline-320 & $64: 150$ & $3930 \pm 65$ & & 1980 B.C. \\
\hline-321 & $64: 149$ & $3940 \pm 68$ & & 1990 B.C. \\
\hline-322 & $108: 87$ & $2857 \pm 54$ & & 907 B.C. \\
\hline-323 & $108: 87$ & $2858 \pm 45$ & & 908 B.C. \\
\hline$-325^{4}$ & $63: 8$ & $1461 \pm 65$ & & A.D. 497 \\
\hline-326 & $64: 151$ & $3450 \pm 65$ & & 1500 B.C. \\
\hline-328 & $64: 151$ & $3220 \pm 64$ & & 1270 B.C. \\
\hline-329 & $64: 151$ & $3260 \pm 63$ & & 1310 B.C. \\
\hline-330 & $64: 151$ & $3360 \pm 50$ & & 1410 B.C. \\
\hline-332 & $64: 151$ & $3140 \pm 61$ & & 1190 B.C. \\
\hline-337 & $64: 151$ & $3030 \pm 61$ & & 1080 B.C. \\
\hline-340 & $64: 151$ & $3320 \pm 57$ & & 1370 B.C. \\
\hline-341 & $64: 151$ & $3010 \pm 56$ & & 1060 B.C. \\
\hline-343 & $64: 157$ & $1210 \pm 54$ & & A.D. 740 \\
\hline-344 & $64: 157$ & $1650 \pm 48$ & & A.D. 300 \\
\hline-345 & $64: 157$ & $8060 \pm 100$ & & 6110 B.C. \\
\hline-346 & $163: 181$ & $\Delta+4.2 \pm 4.5$ & $12.13 \pm 4.10$ & \\
\hline-347 & $108: 101$ & $782 \pm 53$ & & A.D. 1168 \\
\hline-352 & $64: 147$ & $2390 \pm 61$ & & 440 B.C. \\
\hline
\end{tabular}




\begin{tabular}{|c|c|c|c|c|c|}
\hline $\begin{array}{l}\text { Laboratory } \\
\text { number }\end{array}$ & $\begin{array}{l}\text { Published } \\
\text { reference }\end{array}$ & $\begin{array}{l}\text { Original date or } \\
\text { other value }\end{array}$ & $\begin{array}{l}\text { Corrected date } \\
\text { or other value }\end{array}$ & $\partial \mathrm{C}^{14}, \% 0$ & $\begin{array}{c}\text { A.D./ } \\
\text { B.C. date }\end{array}$ \\
\hline-353 & $64: 148$ & $2910 \pm 53$ & & & 960 B.C. \\
\hline-354 & $64: 144$ & $1520 \pm 55$ & & & A.D. 430 \\
\hline-356 & $64: 148$ & $2920 \pm 45$ & & & 970 B.C. \\
\hline-358 & $64: 147$ & $2890 \pm 87$ & & & 940 B.C. \\
\hline-363 & $64: 146$ & $2720 \pm 54$ & & & 770 B.C. \\
\hline-370 & $108: 100$ & $1187 \pm 48$ & & & A.D. 763 \\
\hline-372 & $108: 100$ & $1864 \pm 58$ & & & A.D. 86 \\
\hline-373 & $108: 100$ & $800 \pm 47$ & & & A.D. 1150 \\
\hline-375 & $163: 181$ & $\Delta-5.9 \pm 3.2$ & & $2.00 \pm 2.60$ & \\
\hline-376 & $108: 84$ & $11,166 \pm 107$ & & & 9216 B.C. \\
\hline-377 & $108: 84$ & $9582 \pm 89$ & & & 7632 B.C. \\
\hline-378 & $108: 84$ & $9775 \pm 110$ & & & 7825 B.C. \\
\hline-379 & $108: 84$ & $9655 \pm 84$ & & & 7705 B.C. \\
\hline-380 & $108: 84$ & $8610 \pm 75$ & & & 6660 B.C. \\
\hline-381 & $108: 84$ & $8658 \pm 101$ & & & 6708 B.C. \\
\hline-382 & $108: 84$ & $8956 \pm 103$ & & & 7006 B.C. \\
\hline-384 & $108: 83$ & $3079 \pm 64$ & & & 1129 B.C. \\
\hline-387 & $163: 181$ & $\Delta-0.1 \pm 4.4$ & & $7.67 \pm 4.00$ & \\
\hline-389 & $108: 83$ & $6134 \pm 173$ & & & 4184 B.C. \\
\hline $.396 a$ & $163: 181$ & $\Delta+5.0 \pm 5.5$ & & $13.90 \pm 5.14$ & \\
\hline$-397 a$ & $163: 181$ & $\Delta-4.8 \pm 4.5$ & & $3.00 \pm 4.06$ & \\
\hline-398 & $108: 89$ & $2473 \pm 54$ & & & 523 B.C. \\
\hline-399 & $108: 88$ & $2521 \pm 54$ & & & 571 B.C. \\
\hline-406 & $108: 100$ & $1525 \pm 58$ & & & A.D. 425 \\
\hline-407 & $163: 183$ & $4042 \pm 69$ & & & 2092 B.C. \\
\hline-409 & $108: 94$ & $4175 \pm 223$ & & & 2225 B.C. \\
\hline-4.11 & $163: 183$ & $3672 \pm 66$ & & & 1722 B.C. \\
\hline-413 & $163: 183$ & $3747: 67$ & & & 1797 B.C. \\
\hline-415 & $163: 185$ & $3237 \pm 55$ & & & 1287 B.C. \\
\hline-416 & $163: 183$ & $169 \pm 47$ & & & A.D. 1781 \\
\hline-418 & $108: 86$ & $2899 \pm 49$ & & & 949 B.C. \\
\hline-419 & $108: 86$ & $2880 \pm 45$ & & & 930 B.C. \\
\hline-420 & $108: 89$ & $2347 \pm 54$ & & & 397 B.C. \\
\hline-421 & $108: 86$ & $2913 \pm 56$ & & & 963 B.C. \\
\hline-423 & $108: 86$ & $2896 \pm 51$ & & & 946 B.C. \\
\hline-424 & $108: 87$ & $2816 \pm 55$ & & & 866 B.C. \\
\hline-425 & $108: 87$ & $2872 \pm 62$ & & & 922 B.C. \\
\hline-426 & $108: 82$ & $>41,000$ & & & \\
\hline-427 & $163: 185$ & $2347 \pm 48$ & & & 397 B.C. \\
\hline-428 & $163: 185$ & $1594 \pm 40$ & & & A.D. 356 \\
\hline-429 & $163: 185$ & $2832 \pm 44$ & & & 882 B.C. \\
\hline-430 & $163: 183$ & $2329 \pm 46$ & & & 379 B.C. \\
\hline-431 & $163: 183$ & $656 \pm 40$ & & & A.D. 1294 \\
\hline-433 & $163: 183$ & $627 \pm 56$ & & & A.D. 1323 \\
\hline-434 & $163: 183$ & $3938 \pm 51$ & & & 1988 B.C. \\
\hline-435 & $163: 183$ & $3767 \pm 56$ & & & 1817 B.C. \\
\hline-436 & $163: 183$ & $2277 \pm 42$ & & & 327 B.C. \\
\hline-437 & $108: 87$ & $2841 \pm 63$ & & & 891 B.C. \\
\hline-438 & $108: 90$ & $5990 \pm 81$ & & & 4040 B.C. \\
\hline .439 & $108: 88$ & $2811 \pm 64$ & & & 861 B.C. \\
\hline-440 & $108: 85$ & $2864 \pm 64$ & & & 914 B.C. \\
\hline-442 & 108:91 & $5815 \pm 83$ & & & 3865 B.C. \\
\hline-448 & $163: 182$ & $\Delta+14.8 \pm 5.6$ & & $23.19 \pm 4.09$ & \\
\hline-449 & $163: 182$ & $\vec{\Delta}+21.8 \pm 5.6$ & & $30.25 \pm 4.09$ & \\
\hline-450 & $163: 182$ & $\Delta+23.0 \pm 5.2$ & & $31.47 \pm 3.56$ & \\
\hline-451 & $163: 183$ & $2450 \pm 47$ & & & 500 B.C. \\
\hline-453 & $163: 183$ & $601 \pm 48$ & & & A.D. 1349 \\
\hline-455 & $108: 90$ & $7269 \pm 86$ & & & 5319 B.C. \\
\hline-456 & $108: 94$ & $1583 \pm 58$ & & & A.D. 367 \\
\hline-457 & $108: 83$ & $7184 \pm 84$ & & & 5234 B.C. \\
\hline-458 & $108: 83$ & $7686 \pm 112$ & & & 5736 B.C. \\
\hline
\end{tabular}




\begin{tabular}{|c|c|c|c|c|c|}
\hline $\begin{array}{l}\text { Laboratory } \\
\text { number }\end{array}$ & $\begin{array}{l}\text { Published } \\
\text { reference }\end{array}$ & $\begin{array}{l}\text { Original date or } \\
\text { other value }\end{array}$ & $\begin{array}{l}\text { Corrected date } \\
\text { or other value }\end{array}$ & $\partial \mathrm{C}^{14}, \%$ & $\begin{array}{l}\text { A.D./ } \\
\text { B.C. date }\end{array}$ \\
\hline-459 & $108: 83$ & $8142 \pm 100$ & & & 6192 B.C. \\
\hline-460 & $108: 83$ & $8364 \pm 101$ & & & 64.14 B.C. \\
\hline-461 & $108: 83$ & $2991 \pm 53$ & & & 1041 B.C. \\
\hline-462 & $108: 83$ & $3140 \pm 151$ & & & 1140 B.C. \\
\hline-463 & $108: 85$ & $2643 \pm 63$ & & & 693 B.C. \\
\hline-464 & $108: 85$ & $2690 \pm 62$ & & & 740 B.C. \\
\hline-465 & 108:92 & $7605 \pm 96$ & & & 5655 B.C. \\
\hline-466 & $108: 91$ & $7956 \pm 98$ & & & 6006 B.C. \\
\hline-467 & $108: 92$ & $764.4 \pm 89$ & & & 5694. B.C. \\
\hline-468 & $108: 85$ & $4939 \pm 75$ & & & 2989 B.C. \\
\hline-469 & $108: 85$ & $5169 \pm 64$ & & & 3219 B.C. \\
\hline-472 & $108: 92$ & $3157 \pm 68$ & & & 1207 B.C. \\
\hline-473 & $108: 92$ & $3184 \pm 68$ & & & 1234 B.C. \\
\hline-474 & $108: 92$ & $3099 \pm 185$ & & & 2149 B.C. \\
\hline-475 & $108: 93$ & $3455 \pm 70$ & & & 1505 B.C. \\
\hline-476 & $108: 93$ & $4125 \pm 69$ & & & 2175 B.C. \\
\hline .478 & $108: 94$ & $3740 \pm 64$ & & & 1790 B.C. \\
\hline-481 & $108: 92$ & $3879 \pm 72$ & & & 1929 B.C. \\
\hline-482 & $108: 96$ & $3306 \pm 61$ & & & 1356 B.C. \\
\hline-483 & $108: 96$ & $3344 \pm 61$ & & & 1394 B.C. \\
\hline .485 & $108: 96$ & $3271 \pm 48$ & & & 1321 B.C. \\
\hline-486 & $108: 96$ & $3373 \pm 58$ & & & 1423 B.C. \\
\hline-487 & $108: 96$ & $3284 \pm 61$ & & & 1334 B.C. \\
\hline-488 & $108: 96$ & $3361 \pm 70$ & & & 1411 B.C. \\
\hline .489 & $108: 96$ & $3424 \pm 62$ & & & 1474 B.C. \\
\hline -4.91 & $163: 182$ & $\Delta+18.4 \pm 5.6$ & & $25.60 \pm 4.09$ & \\
\hline-492 & $163: 183$ & $2448 \pm 47$ & & & 498 B.C. \\
\hline-493 & $163: 183$ & $3002 \pm 57$ & & & 1052 B.C. \\
\hline-494 & $163: 181$ & $\Delta+11.6 \pm 4.5$ & & $17.92 \pm 4.12$ & \\
\hline-495 & $163: 181$ & $\Delta+10.6 \pm 4.4$ & & $17.95 \pm 4.02$ & \\
\hline-496 & $163: 181$ & $\Delta-4.9 \pm 3.7$ & & $2.97 \pm 3.21$ & \\
\hline-497 & $163: 181$ & $\Delta-0.1 \pm 4.4$ & & $9.05 \pm 4.03$ & \\
\hline-498 & $163: 181$ & $\Delta+4.8 \pm 4.5$ & & $12.73 \pm 4.06$ & \\
\hline-499 & $163: 181$ & $\Delta+9.6 \pm 5.2$ & & $15.10 \pm 4.83$ & \\
\hline-500 & $108: 96$ & $3496 \pm 56$ & & & 1546 B.C. \\
\hline-502 & $108: 90$ & $6895 \pm 83$ & & & 4945 B.C. \\
\hline-503 & $108: 90$ & $5986 \pm 87$ & & & 4036 B.C. \\
\hline-504 & $108: 89$ & $5518 \pm 81$ & & & 3568 B.C. \\
\hline-505 & $108: 89$ & $5638 \pm 85$ & & & 3688 B.C. \\
\hline-506 & 108:91 & $1773 \pm 62$ & & & A.D. 177 \\
\hline-507 & $108: 91$ & $2850 \pm 59$ & & & 900 B.C. \\
\hline-508 & $108: 91$ & $3996 \pm 242$ & & & 2046 B.C. \\
\hline-509 & $108: 91$ & $3917 \pm 156$ & & & 1967 B.C. \\
\hline-511 & $108: 99$ & $1345 \pm 118$ & & & 605 B.C. \\
\hline-512 & $108: 97$ & $1058 \pm 52$ & & & A.D. 892 \\
\hline-513 & $108: 98$ & $1968 \pm 62$ & & & 18 B.C. \\
\hline-515 & $108: 97$ & $2014 \pm 62$ & & & 64 B.C. \\
\hline-516 & $108: 98$ & $2408 \pm 214$ & & & 458 B.C. \\
\hline-517 & 108:98 & $2302 \pm 125$ & & & 352 B.C. \\
\hline-518 & $108: 98$ & $2195 \pm 64$ & & & 245 B.C. \\
\hline-519 & $108: 96$ & $9028 \pm 120$ & & & 7078 B.C. \\
\hline-521 & $108: 96$ & $9720 \pm 128$ & & & 7770 B.C. \\
\hline-522 & $108: 94$ & $4378 \pm 196$ & & & 2428 B.C. \\
\hline-523 & $108: 94$ & $4029 \pm 74$ & & & 2079 B.C. \\
\hline-524 & $108: 93$ & $5474 \pm 83$ & & & 3524 B.C. \\
\hline-525 & $108: 93$ & $3193 \pm 69$ & & & 1243 B.C. \\
\hline-526 & $108: 93$ & $3136 \pm 68$ & & & 1186 B.C. \\
\hline-527 & $108: 93$ & $2515 \pm 58$ & & & 565 B.C. \\
\hline-528 & $108: 93$ & $2878 \pm 65$ & & & 928 B.C. \\
\hline-529 & $108: 93$ & $3868 \pm 72$ & & & 1918 B.C. \\
\hline-530 & $108: 85$ & $4672 \pm 74$ & & & 2722 B.C. \\
\hline
\end{tabular}


Radiocarbon Measurements:

\begin{tabular}{|c|c|c|c|c|c|}
\hline $\begin{array}{c}\text { Laboratory } \\
\text { number }\end{array}$ & $\begin{array}{c}\text { Published } \\
\text { reference }\end{array}$ & $\begin{array}{l}\text { Original date or } \\
\quad \text { other value }\end{array}$ & $\begin{array}{l}\text { Corrected date } \\
\text { or other value }\end{array}$ & $\partial \mathrm{C}^{14}, \%$ & $\begin{array}{l}\text { A.D./ } \\
\text { B.C. date }\end{array}$ \\
\hline-531 & $108: 95$ & $295 \pm 192$ & & & A.D. 1655 \\
\hline-532 & $108: 95$ & $1653 \pm 61$ & & & A.D. 297 \\
\hline-533 & $108: 95$ & $778 \pm 133$ & & & A.D. 1172 \\
\hline-534 & $108: 95$ & $1866 \pm 62$ & & & A.D. 84 \\
\hline-536 & $164: 198$ & $4056 \pm 73$ & & & 2106 B.C. \\
\hline-538 & $164: 198$ & $4071 \pm 73$ & & & 2121 B.C. \\
\hline-540 & $108: 97$ & $4490 \pm 136$ & & & 2540 B.C. \\
\hline-541 & $108: 97$ & $4487 \pm 76$ & & & 2537 B.C. \\
\hline$-543 a$ & $163: 181$ & $\Delta+5.1 \pm 3.6$ & & $16.20 \pm 3.09$ & \\
\hline-545 & $163: 181$ & $\Delta+16.5 \pm 3.9$ & & $24.52 \pm 3.42$ & \\
\hline-549 & $163: 181$ & $\Delta-4.4 \pm 4.9$ & & $3.48 \pm 4.51$ & \\
\hline-551 & $163: 181$ & $\Delta+1.7 \pm 4.3$ & & $9.59 \pm 3.81$ & \\
\hline$-558^{8}$ & $108: 97$ & $1318 \pm 59$ & c & & A.D. 632 \\
\hline-576 & $108: 88$ & $2681 \pm 69$ & & & 731 B.C. \\
\hline-577 & $108: 88$ & $2779 \pm 69$ & & & 829 B.C. \\
\hline-579 & $164: 197$ & $2602 \pm 45$ & & & 652 B.C. \\
\hline-582 & $108: 89$ & $2294 \pm 60$ & & & 344 B.C. \\
\hline-584 & $164: 188$ & $7027 \pm 83$ & & & 5077 B.C. \\
\hline-585 & $164: 188$ & $8064 \pm 78$ & & & 6114 B.C. \\
\hline-586 & $164: 198$ & $3905 \pm 67$ & & & 1955 B.C. \\
\hline .587 & $164: 198$ & $4307 \pm 69$ & & & 2357 B.C. \\
\hline$-588^{8}$ & $164: 198$ & $4371 \pm 69$ & & & 2421 B.C. \\
\hline$-589 \mathrm{~b}$ & $163: 181$ & $\Delta-5.2 \pm 4.6$ & & $3.59 \pm 4.18$ & \\
\hline-600 & $163: 183$ & $4006 \pm 52$ & & & 2056 B.C. \\
\hline-602 & $163: 183$ & $3106 \pm 42$ & & & 1156 B.C. \\
\hline-603 & $163: 185$ & $1952 \pm 36$ & & & 2 B.C. \\
\hline-604 & $163: 185$ & $2602 \pm 35$ & & & 652 B.C. \\
\hline-605 & $163: 185$ & $3225 \pm 47$ & & & 1275 B.C. \\
\hline-606 & $163: 181$ & $\Delta-4.0 \pm 4.5$ & & $1.43 \pm 4.13$ & \\
\hline-607 & $163: 181$ & $\Delta-9.4 \pm 4.4$ & & $-2.15 \pm 4.00$ & \\
\hline-608 & $163: 181$ & $\Delta-9.9 \pm 5.3$ & & $-2.06 \pm 4.93$ & \\
\hline-609 & $163: 181$ & $\Delta+0.1 \pm 5.3$ & & $5.48 \pm 4.97$ & \\
\hline-610 & $163: 181$ & $\Delta+3.7 \pm 5.5$ & & $7.74 \pm 5.21$ & \\
\hline-630 & $163: 181$ & $\Delta+3.8 \pm 4.6$ & & $12: 67 \pm 4.22$ & \\
\hline-631 & $163: 181$ & $\Delta+7.9 \pm 4.6$ & & $16.89 \pm 4.21$ & \\
\hline-632 & $163: 181$ & $\Delta-9.3 \pm 6.1$ & & $-1.53 \pm 5.74$ & \\
\hline-633 & $163: 181$ & $\Delta+4.1 \pm 4.6$ & & $9.55 \pm 4.22$ & \\
\hline-634 & $163: 181$ & $\Delta-0.3 \pm 4.6$ & & $8.55 \pm 4.19$ & \\
\hline-636 & $163: 182$ & $\Delta-0.1 \pm 5.6$ & & $8.78 \pm 4.06$ & \\
\hline$-638 a$ & $163: 181$ & $\bar{\Delta}-0.7 \pm 4.8$ & & $7: 20 \pm 4.40$ & \\
\hline-643 & $163: 181$ & $\Delta+2.6 \pm 6.6$ & & $9.84 \pm 6.29$ & \\
\hline-644 & $163: 181$ & $\Delta+5.1 \pm 5.0$ & & $12.96 \pm 4.72$ & \\
\hline-645 & $163: 181$ & $\Delta-1.1 \pm 6.1$ & & $6.71 \pm 5.83$ & \\
\hline-646 & $163: 181$ & $\Delta+1.6 \pm 5.4$ & & $12.33 \pm 5.09$ & \\
\hline-647 & $163: 181$ & $\Delta+20.4 \pm 4.5$ & & $28.38 \pm 4.45$ & \\
\hline-656 & $163: 182$ & $\Delta+14.0 \pm 5.9$ & & $24.16 \pm 4.46$ & \\
\hline-661 & $163: 182$ & $\Delta+28.9 \pm 5.7$ & & $36.23 \pm 4.18$ & \\
\hline-663 & $163: 182$ & $\Delta+22.8 \pm 5.7$ & & $28.78 \pm 4.19$ & \\
\hline-665 & $163: 182$ & $\Delta+23.1 \pm 5.7$ & & $42.81 \pm 4.25$ & \\
\hline-667 & $163: 182$ & $\Delta+22.7 \pm 5.6$ & & $28.18 \pm 4.09$ & \\
\hline-668 & $163: 182$ & $\Delta+23.4 \pm 5.3$ & & $32.36 \pm 3.73$ & \\
\hline-700 & $163: 181$ & $\Delta+3.7 \pm 4.5$ & & $12.80 \pm 4.09$ & \\
\hline .701 & $163: 181$ & $\Delta-4.2 \pm 4.5$ & & $4.68 \pm 4.11$ & \\
\hline-702 & $163: 181$ & $\Delta+4.6 \pm 4.8$ & & $15: 39 \pm 4.37$ & \\
\hline .703 & $163: 181$ & $\Delta-4.9 \pm 4.3$ & & $2.15 \pm 3.88$ & \\
\hline-712 & $164: 197$ & $2929 \pm 66$ & & & 979 B.C. \\
\hline-714 & $164: 196$ & $3045 \pm 66$ & & & 1095 B.C. \\
\hline .715 & $164: 197$ & $2888 \pm 127$ & & & 938 B.C. \\
\hline-716 & $164: 197$ & $2964 \pm 59$ & & & 1014 B.C. \\
\hline-717 & $164: 196$ & $3111 \pm 59$ & & & 1161 B.C. \\
\hline-718 & $164: 196$ & $3087 \pm 59$ & & & 1137 B.C. \\
\hline
\end{tabular}




\begin{tabular}{|c|c|c|c|c|c|}
\hline $\begin{array}{c}\text { Laboratory } \\
\text { number }\end{array}$ & $\begin{array}{c}\text { Published } \\
\text { reference }\end{array}$ & $\begin{array}{l}\text { Original date or } \\
\quad \text { other value }\end{array}$ & $\begin{array}{l}\text { Corrected date } \\
\text { or other value }\end{array}$ & $\partial \mathrm{C}^{14}, \% 0$ & $\begin{array}{l}\text { A.D./ } \\
\text { B.C. date }\end{array}$ \\
\hline-720 & $164: 196$ & $2981 \pm 58$ & & & 1031 B.C. \\
\hline .721 & $164: 196$ & $6195 \pm 70$ & & & 4245 B.C. \\
\hline-722 & $164: 196$ & $6126 \pm 70$ & & & 4176 B.C. \\
\hline .723 & $164: 197$ & $3053 \pm 59$ & & & 1103 B.C. \\
\hline-724 & $164: 190$ & $3959 \pm 59$ & & & 2009 B.C. \\
\hline-725 & $164: 195$ & $4550 \pm 60$ & & & 2600 B.C. \\
\hline .726 & $164: 196$ & $2980 \pm 50$ & & & 1030 B.C. \\
\hline-738 & $163: 182$ & $\Delta+0.8 \pm 6.3$ & & $4.26 \pm 5.20$ & \\
\hline-760 & $164: 193$ & $2970 \pm 50$ & & & 1020 B.C. \\
\hline .769 & $164: 192$ & $7505 \pm 93$ & & & 5555 B.C. \\
\hline-770 & $164: 192$ & $7912 \pm 94$ & & & 5962 B.C. \\
\hline-772 & $164: 192$ & $7572 \pm 91$ & & & 5622 B.C. \\
\hline .775 & $164: 192$ & $8037 \pm 96$ & & & 6087 B.C. \\
\hline-776 & $164: 192$ & $7640 \pm 91$ & & & 5690 B.C. \\
\hline-777 & $164: 191$ & $7704 \pm 91$ & & & 5754 B.C. \\
\hline-778 & $164: 191$ & $7538 \pm 89$ & & & 5588 B.C. \\
\hline .779 & $164: 191$ & $8190 \pm 99$ & & & 6240 B.C. \\
\hline-780 & $163: 182$ & $\Delta+28.4 \pm 5.7$ & & $35.66 \pm 4.11$ & \\
\hline .781 & $164: 192$ & $7524 \pm 90$ & & & 5574 B.C. \\
\hline .782 & $164: 191$ & $8092 \pm 98$ & & & 6142 B.C. \\
\hline-785 & $163: 184$ & $2147 \pm 46$ & $\Delta+0.8 \pm 4.5$ & $11.01 \pm 4.34$ & 197 B.C. \\
\hline-786 & $163: 184$ & $1576 \pm 43$ & $\Delta+6.6 \pm 4.4$ & $13.77 \pm 4.22$ & A.D. 374 \\
\hline-787 & $163: 184$ & $1357 \pm 66$ & $\Delta-8.2 \pm 7.1$ & $0.78 \pm 6.96$ & A.D. 607 \\
\hline-788 & $164: 194$ & $2691 \pm 47$ & & & 741 B.C. \\
\hline-789 & $164: 193$ & $6980 \pm 79$ & & & 5030 B.C. \\
\hline-790 & $164: 193$ & $6830 \pm 78$ & & & 4880 B.C. \\
\hline-791 & $164: 193$ & $6755 \pm 80$ & & & 4805 B.C. \\
\hline .792 & $164: 193$ & $6670 \pm 76$ & & & 4720 B.C. \\
\hline-793 & $164: 193$ & $6254 \pm 78$ & & & 4304 B.C. \\
\hline-794 & $164: 193$ & $7033 \pm 89$ & & & 5083 B.C. \\
\hline .795 & $164: 193$ & $6832 \pm 78$ & & & 4882 B.C. \\
\hline .796 & $164: 192$ & $7521 \pm 77$ & & & 5571 B.C. \\
\hline-797 & $164: 192$ & $7629 \pm 90$ & & & 5679 B.C. \\
\hline-798 & $164: 188$ & $4145 \pm 59$ & & & 2195 B.C. \\
\hline .799 & $164: 189$ & $8472 \pm 102$ & & & 6522 B.C. \\
\hline-800 & $164: 189$ & $4157 \pm 62$ & & & 2207 B.C. \\
\hline-801 & $164: 189$ & $4590 \pm 65$ & & & 2640 B.C. \\
\hline-803 & $164: 188$ & $4221 \pm 53$ & & & 2271 B.C. \\
\hline-804 & $164: 189$ & $4095 \pm 52$ & & & 2145 B.C. \\
\hline-805 & $164: 189$ & $4006 \pm 62$ & & & 2056 B.C. \\
\hline-806 & $164: 189$ & $4345 \pm 66$ & & & 2395 B.C. \\
\hline-807 & $164: 189$ & $4090 \pm 64$ & & & 2140 B.C. \\
\hline-809 & $164: 189$ & $3945 \pm 62$ & & & 1995 B.C. \\
\hline-810 & $164: 188$ & $4074 \pm 64$ & & & 2124 B.C. \\
\hline-811 & $163: 184$ & $2367 \pm 46$ & $\Delta 13.9 \pm 4.4$ & $23.08 \pm 4.25$ & 417 B.C. \\
\hline-812 & $163: 181$ & $\Delta-3.2 \pm 4.1$ & & $4.92 \pm 3.73$ & \\
\hline .813 & $163: 181$ & $\Delta-2.4 \pm 5.1$ & & $5.46 \pm 4.73$ & \\
\hline-815 & $163: 182$ & $\Delta-3.1 \pm 5.6$ & & $4.11 \pm 4.08$ & \\
\hline-818 & $163: 182$ & $\Delta+2.8 \pm 4.5$ & & $8.25 \pm 4.27$ & \\
\hline-819 & $164: 189$ & $4272 \pm 65$ & & & 2322 B.C. \\
\hline-820 & $164: 189$ & $4090 \pm 62$ & & & 2140 B.C. \\
\hline-821 & $163: 182$ & $\Delta-3.2 \pm 4.6$ & & $10.50 \pm 4.33$ & \\
\hline-824 & $164: 191$ & $3744 \pm 61$ & & & 1794 B.C. \\
\hline-825 & $164: 191$ & $4540 \pm 56$ & & & 2590 B.C. \\
\hline-826 & $164: 191$ & $4200 \pm 58$ & & & 2250 B.C. \\
\hline-827 & $164: 192$ & $7579 \pm 86$ & & & 5629 B.C. \\
\hline-829 & $164: 195$ & $2596 \pm 56$ & & & 646 B.C. \\
\hline-830 & $164: 195$ & $2572 \pm 59$ & & & 622 B.C. \\
\hline-831 & $164: 195$ & $2542 \pm 46$ & & & 592 B.C. \\
\hline-832 & $164: 195$ & $2406 \pm 52$ & & & 456 B.C. \\
\hline-833 & $164: 195$ & $2537 \pm 52$ & & & 587 B.C. \\
\hline
\end{tabular}




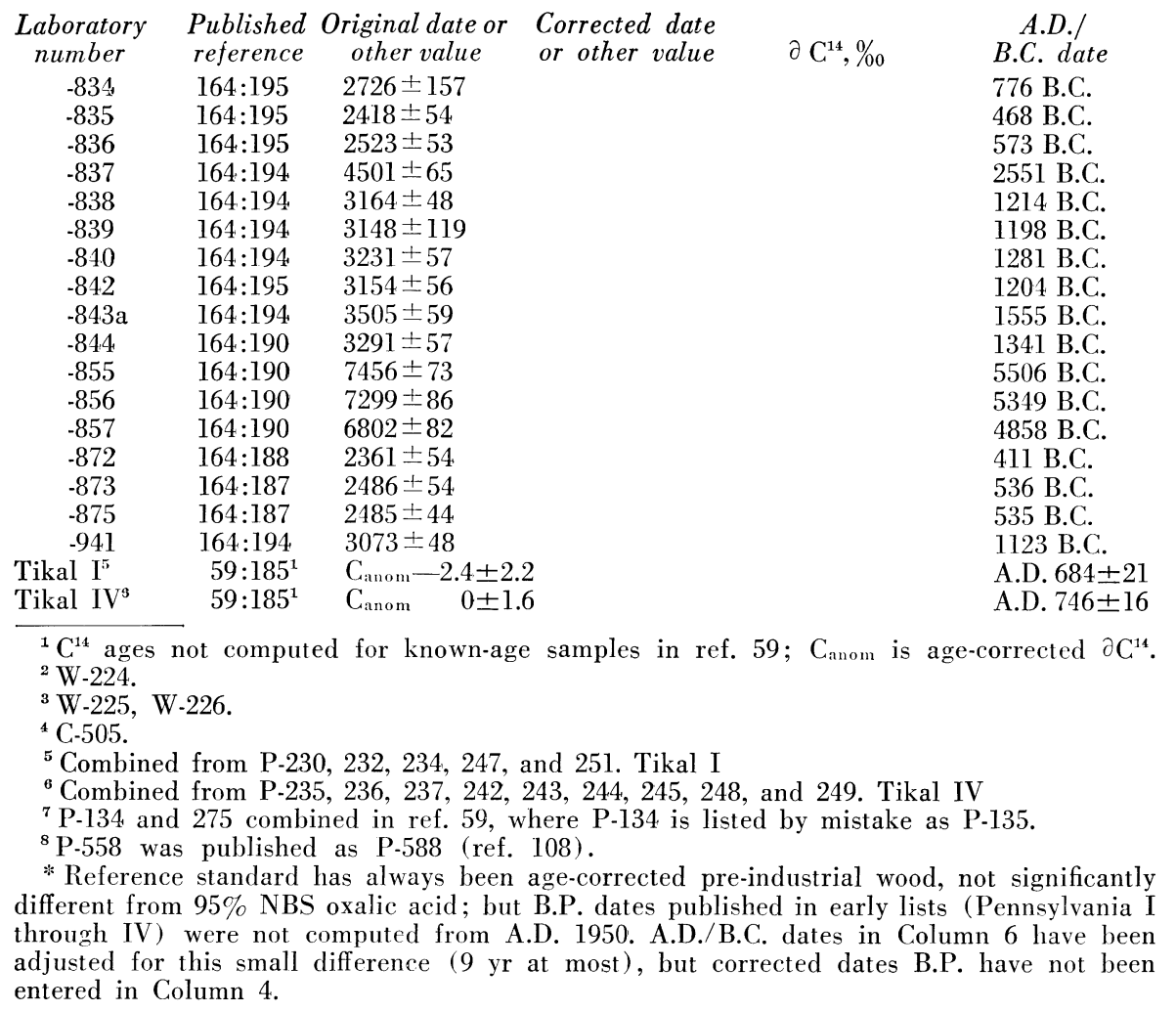

\section{Pisa, Lahoratoria de Geologia Nucleare dell'Universita ${ }^{1}$}

\begin{tabular}{|c|c|c|c|c|c|}
\hline $\begin{array}{c}\text { Laboratory } \\
\text { number }\end{array}$ & $\begin{array}{l}\text { Published } \\
\text { reference }\end{array}$ & $\begin{array}{l}\text { Original date or } \\
\text { other value }\end{array}$ & $\begin{array}{l}\text { Corrected date } \\
\text { or other value }\end{array}$ & $\partial \mathrm{C}^{14}, \%$ & $\begin{array}{l}\text { A.D./ } \\
\text { B.C. date }\end{array}$ \\
\hline Pi-1 & $69: 103$ & $2318 \pm 105$ & & & 368 B.C. \\
\hline$-3 \mathrm{~A}$ & $68: 108$ & $1863 \pm 135$ & & & A.D. 87 \\
\hline$-3 B$ & $68: 108$ & $2160 \pm 145$ & & & 210 B.C. \\
\hline-4 & $68: 106$ & $5534 \pm 144$ & & & 3584 B.C. \\
\hline-5 & $68: 108$ & $2908 \pm 153$ & & & 958 B.C. \\
\hline-6 & $68: 108$ & $10,178 \pm 400$ & & & 8228 B.C. \\
\hline$-7 \mathrm{~A}$ & $68: 109$ & $26,364 \pm 1405$ & & & 24,414 B.C. \\
\hline$-7 \mathrm{~B}$ & $68: 109$ & $6892 \pm 260$ & & & 4942 B.C. \\
\hline-10 & $68: 106$ & $8619 \pm 200$ & & & 6669 B.C. \\
\hline-13 & $68: 110$ & $3767 \pm 115$ & & & 1817 B.C. \\
\hline-15 & $68: 107$ & $3475 \pm 86$ & & & 1525 B.C. \\
\hline$-15 \mathrm{~A}$ & $68: 107$ & $3470 \pm 120$ & & & 1520 B.B. \\
\hline$-15 B$ & $68: 107$ & $3480 \pm 120$ & & & 1530 B.C. \\
\hline-18 & $68: 107$ & $2176 \pm 105$ & & & 226 B.C. \\
\hline-19 & $68: 109$ & $892 \pm 100$ & & & A.D. 1058 \\
\hline-20 & $68: 109$ & $1968 \pm 110$ & & & 18 B.C. \\
\hline-21 & $68: 110$ & $6659 \pm 153$ & & & 4709 B.C. \\
\hline-22 & $68: 108$ & $1847 \pm 105$ & & & A.D. 103 \\
\hline-23 & $68: 106$ & $12,619 \pm 410$ & & & 10.669 B.C. \\
\hline-25 & $68: 107$ & $3333 \pm 115$ & & & 1383 B.C. \\
\hline-26 & $68: 107$ & $3100 \pm 113$ & & & 1150 B.C. \\
\hline-28 & $68: 106$ & $5398 \pm 145$ & & & 3448 B.C. \\
\hline
\end{tabular}

